\title{
Distinct Excitonic Circular Dichroism between Wurtzite and Zincblende CdSe Nanoplatelets
}

\author{
Xiaoqing Gao, ${ }^{\dagger \neq}$ Xiuwen Zhang, ${ }^{\ddagger \odot}$ Luyang Zhao, ${ }^{\S}$ Pu Huang, ${ }^{\ddagger}$ Bing Han, ${ }^{\dagger}$ Jiawei Lv, ${ }^{\dagger}$ Xueying Qiu, \\ Su-Huai Wei," and Zhiyong Tang*, ${ }^{\dagger} \odot$
}

${ }^{\dagger}$ CAS Key Laboratory of Nanosystem and Hierarchical Fabrication, CAS Center for Excellence in Nanoscience, National Center for Nanoscience and Technology, Beijing 100190, People's Public of China

"Shenzhen Key Laboratory of Flexible Memory Materials and Devices, College of Electronic Science and Technology, Shenzhen University, Shenzhen, Guangdong 518060, People's Public of China

${ }^{\S}$ National Key Laboratory of Biochemical Engineering, Institute of Process Engineering, Chinese Academy of Science, Beijing 100190, People's Republic of China?

"Beijing Computational Science Research Center, Beijing 100094, People’s Public of China

Supporting Information

ABSTRACT: Nanocrystals (NCs) with identical components and sizes but different crystal structures could not be distinguished by conventional absorption and emission spectra. Herein, we find that circular dichroism (CD) spectroscopy can easily distinguish the CdSe nanoplatelets (NPLs) with different crystal structures of wurtzite (WZ) and zincblende (ZB) with the help of chiral L- or D-cysteine ligands. In particular, the $\mathrm{CD}$ signs of the first excitonic transitions in $\mathrm{WZ}$ and $\mathrm{ZB}$ NPLs capped by the same chiral cysteine are opposite. Theoretic calculation supports the viewpoint of different crystal structures and surfaces arrangements between WZ and ZB NPLs contributing to this significant phenomenon. The $C D$ peaks appearing at the first excitonic transition band of WZ or ZB CdSe NPLs are clearly assigned to the different transition polarizations along $4 \mathrm{p}_{(x, y, z), \mathrm{Se}} \rightarrow 5 \mathrm{~s}_{\mathrm{Cd}}$ or $4 \mathrm{p}_{(x, y), \mathrm{Se}} \rightarrow$

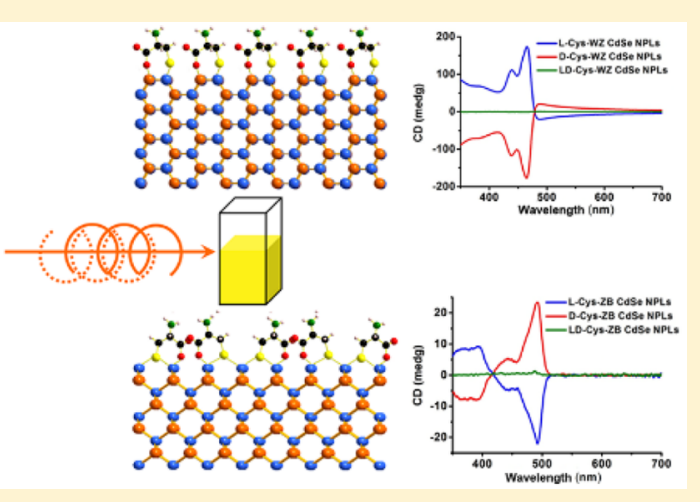
$5 s_{\mathrm{Cd}}$. This work not only provides a deep insight into the origin of the optical activity inside chiral semiconductor nanomaterials but also proposes the design principle of chiral semiconductor nanocrystals with high optic activity.

KEYWORDS: circular dichroism spectrum, optical activity, CdSe nanoplatelets, wurtzite, zincblende, excitonic transition

\begin{abstract}
A s a new member in the chiral family, optically active colloidal semiconductor nanocrystals (NCs) constructed with chiral molecules as stabilizers have recently received intensive research interest due to their quantum-confinementdependent optical activity. ${ }^{1-8}$ Many efforts have been put to improve their fundamental understanding as well as practical applications, and both have achieved considerable progress. $^{7-10}$

The typical method used to characterize the optical activity of chiral semiconductor NCs is circular dichroism (CD) spectroscopy. Conventionally, the analysis methods involved with CD spectra of chiral molecules can be categorized into below three types: ${ }^{11} \mathrm{I}$, empirical, a qualitative analysis of change in the spectra; II, ab initio, the calculation using complete molecular wave functions directly from the Rosenfeld equation; and III, chromophoric, a molecule divided into separate chromophores with corresponding calculation. The development of $\mathrm{CD}$ spectrum analysis on chiral semiconductor NCs follows the same tendency. At the beginning, the scientists just described the change of the obtained CD spectrum of this system as type $\mathrm{I}^{1,3,12,13}$
\end{abstract}

Next comes type II. The huge number of the atoms in this system increases the difficulty of ab initio calculation. In spite of this, many scientists devote efforts to getting a clear understanding of the interesting optical activity of chiral semiconductor NCs by adopting this method. The reported theoretical calculations might be further divided into two types: interface analysis or treating the chiral $\mathrm{NC}$ as a whole.

As for interface analysis, Elliott et al. applied the density functional theory (DFT) to treat the chiral penicillaminestabilized CdS NCs and revealed that penicillamine strongly distorted surface $\mathrm{Cd}$ (and, thus, led to the transmission of an enantiomeric structure to the surface layers). ${ }^{14}$ Our group analyzed the cysteine-stabilized $\mathrm{CdTe} \mathrm{NCs}$ by quantum mechanical calculation with the software package Spartan O4 and demonstrated the new chiral $\mathrm{Cd}$ centers formed on the surface of CdTe. ${ }^{2}$ Balaz's group reported that the $\mathrm{CD}$ signs of $\mathrm{N}$-acetyl-L-cysteine-CdSe NCs and L-homocysteine-CdSe NCs

Received: March 13, 2018

Revised: October 6, 2018

Published: October 12, 2018 
were inverted. In their work, DFT and time-dependent DFT (TDDFT) were used to support the proposal that the chiral inversion originated from different binding arrangements of the chiral molecules on the surfaces of NCs. ${ }^{15}$ Kotov's group used DFT calculation to indicate that the covalent attachment of Land $\mathrm{D}$-cysteine moieties to the edges of graphene quantum dots (GQDs) resulted in their helical buckling. ${ }^{16}$

In regard to treating the chiral NC as a whole, Balaz and coworkers employed TDDFT with a simplified model of a chiral cluster with tens of atoms to analyze the semiconductor NCs. They pointed out that the induced chirality was consistent with the hybridization of the highest occupied molecular orbitals of CdSe with those of the chiral ligands. ${ }^{17}$ Very recently, Kotov and co-workers used the simplified TDDFT method proposed by Grimme ${ }^{18,19}$ to acquire the $\mathrm{CD}$ spectrum of chiral $\mathrm{Co}_{3} \mathrm{O}_{4}$ NCs. ${ }^{10}$ Here, the structure of NCs was simplified to reduce the computing difficulty.

Altogether, most current analyses on the CD spectra of chiral NC system are based on the information obtained by TDDFT calculation. However, the vast atoms of this system increase the difficulty. To solve this challenge, our group introduced the chromophoric analysis (type III), by which the NC could be treated as a chromophore. ${ }^{20}$ Actually, chromophoric analysis has been proved to be a powerful method for treating the huge chiral organic molecules. ${ }^{11}$ With the help of the nondegenerate coupled-oscillator model (NDCO) arising from chromophoric analysis, NCs do not need to be simplified as the clusters that likely lose their periodic structures. At the same time, the exploratory applications based on the excellent optical properties of chiral semiconductor NCs include the detection of chiral drugs and amino acids, ${ }^{21-25}$ in vivo targeted imaging, ${ }^{26}$ cytotoxicity mediation and cell labels, ${ }^{27,28}$ asymmetric catalysis, ${ }^{29}$ enantiomeric detection and separation, ${ }^{10,30}$ detection of metal ions, ${ }^{31}$ spin selectivity, ${ }^{32,33}$ and so on.

It is well-known that the magnetic, electrical, and optical properties of nanomaterials are determined by their size, shape, and crystal structure. ${ }^{34,35}$ Recent research on the chiral semiconductor NCs likewise supports the size- and shapedependent optical activity. ${ }^{3,17,20}$ The chiral quantum dots (QDs) stabilized by amino acid are reported to exhibit a quantum-confinement-based optical activity: namely, the CD peaks of the excitonic transitions red-shift as the size increases. ${ }^{3,17}$ Furthermore, the anisotropic $g$-factor at the first excitonic transition shows a 10 -fold increase as the shape changed from spherical QDs to one-dimensional quantum rods (QRs). ${ }^{20}$ Nevertheless, the influence of the crystal structure on the optical activity of the chiral semiconductor nanomaterials remains unexplored.

In this work, we focus on the chiral biomolecule stabilized wurtzite (WZ) and zincblende (ZB) CdSe nanoplateles (NPLs) to study the crystal structure effect. These systems are chosen for a couple of reasons. On the one hand, pure $\mathrm{WZ}$ and ZB CdSe NPLs are easily obtained by controlling the reaction temperature and precursors. ${ }^{36}$ On the other hand, CdSe NPLs have conspicuous properties such as narrow fluorescent spectrum, ${ }^{37,38}$ large exciton and biexciton binding energies and two-photon-absorption cross-sections, ${ }^{39-41}$ fast and efficient fluorescence resonance energy transfer, ${ }^{42}$ weak coupling of carriers to acoustic phonons, ${ }^{43}$ and so on. ${ }^{44}, 45$ However, the research of their chiral optical activity is rare. Hence, the WZ and ZB CdSe NPLs with the same thickness are fabricated to investigate the influence of the crystal structure on the optical activity of chiral semiconductor NCs.

WZ and ZB CdSe NPLs with the same thickness of about $1.4 \mathrm{~nm}$ were selected as the research objects to avoid the influence of the thickness-dependent quantum effect. The demonstration of the $1.4 \mathrm{~nm}$ thickness will be offered later. Assynthesized WZ CdSe NPLs stabilized by octylamine (OAm) and oleylamine (OLAm) [ noted as (OAm, OLAm)-WZ CdSe NPLs] were dissolved into chloroform, while ZB CdSe NPLs with oleic acid (OA) as stabilizers (noted as OA-ZB CdSe NPLs) were dissolved into hexane. In the subsequent ligandexchange process, chiral amino acid, L- or D-cysteine (or LDcysteine, the racemes), was added onto the surface of $\mathrm{WZ}$ and ZB CdSe NPLs through a strong Cd-S bond. The NPLs after exchanged with $\mathrm{L}$-cysteine were fully dissolved into water (Figure S1) and noted as L-Cys-WZ CdSe NPLs and L-Cys-ZB CdSe NPLs (similar phenomena happened for the D- or L,D- or LD-cysteine-stabilized NPLs). Such ligand exchange has a slight effect on the size and no influence on the crystal structure of both WZ and ZB CdSe NPLs (Figures 1 and S2-S4). $\mathrm{H}^{1-}$ NMR spectra further verify complete ligand exchange (Figure S5), and there are no residual OAm, OLAm, or OA molecules on the surface of NPLs.

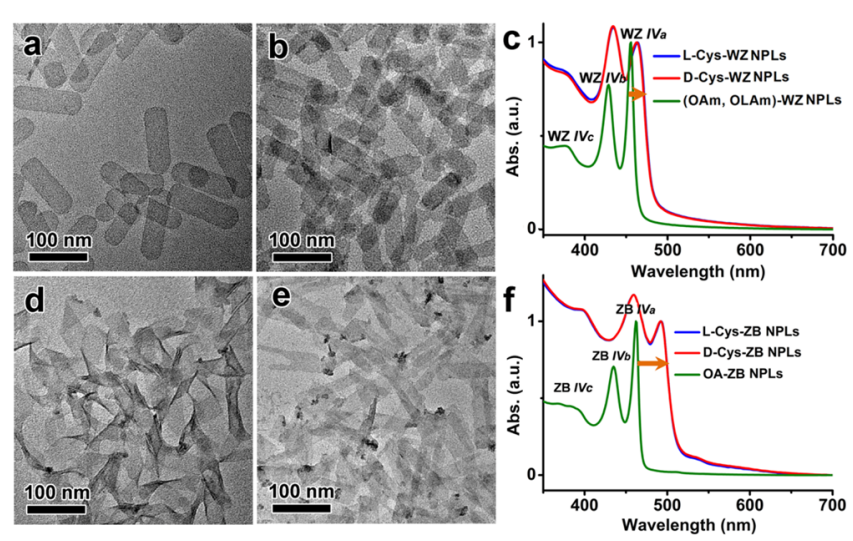

Figure 1. Transmission electron microscope (TEM) images and UVvis absorption spectra of WZ and ZB CdSe NPLs before and after ligand exchange. (a) TEM image of (OAm, OLAm)-WZ CdSe NPLs. (b) TEM image of L-Cys-WZ CdSe NPLs. (c) UV-vis absorption spectra of WZ CdSe NPLs before and after ligand exchange. (d) TEM image of OA-ZB CdSe NPLs. (e) TEM image of L-Cys-ZB CdSe NPLs. (f) UV-vis absorption spectra of ZB CdSe NPLs before and after ligand exchange. The peaks in the green curves with gradual decrease of the wavelength are noted as WZ $I V_{a}, \mathrm{WZ} I V_{b}$, and WZ IV in WZ CdSe NPLs and ZB $I V_{a}, \mathrm{ZB} I V_{b}$, and ZB $I V_{c}$ in ZB CdSe NPLs.

The excitonic transitions in both (OAm, OLAm)-WZ CdSe NPLs and OA-ZB CdSe NPLs are affected by the quantum confinement effect because the thickness of $1.4 \mathrm{~nm}$ is much smaller than the bulk exciton Bohr radius of $5.6 \mathrm{~nm}{ }^{46}$ Absorption spectra manifest the quantum-well characteristics of the electronic band structure with two sharp Lorenz peaks (green curves in Figure 1c,f). The peaks with incremental transition energy correspond to the first heavy hole-first electron (1hh-1e, peaks WZ $I V_{a}$ and $\mathrm{ZB} I V_{a}$ ), the first light hole-first electron (1lh-1e, peaks $\mathrm{WZ} I V_{b}$ and $\mathrm{ZB} I V_{b}$ ), and the first spin orbital coupling-first electron (1SO-1e, peaks WZ $I V_{c}$ and $\left.\mathrm{ZB} I V_{c}\right)$ transitions, respectively. ${ }^{47}$ The transition of $1 \mathrm{hh}-$ 1e locates at $456 \mathrm{~nm}(2.73 \mathrm{eV})$ for (OAm, OLAm)-WZ CdSe NPLs and $462 \mathrm{~nm}(2.69 \mathrm{eV}$ ) for OA-ZB CdSe NPLs (Table 
S1). The slightly higher transition energy of WZ NPLs compared with those of $\mathrm{ZB}$ is coincident with the difference of about $0.1 \mathrm{eV}$ in band gap between the bulk $\mathrm{WZ}$ and $\mathrm{ZB}$ $\mathrm{CdSe}^{48}$ The ligand exchange with $\mathrm{L}$ - or D-cysteine changes the stabilizers on the surface of WZ and ZB NPLs. Thereafter, the 1hh-1e transition red-shifts $8 \mathrm{~nm}(-0.06 \mathrm{eV})$ in WZ CdSe NPLs and $29 \mathrm{~nm}(-0.16 \mathrm{eV})$ in ZB CdSe NPLs (orange arrows in Figure 1c,f and Table S1), and the reason will be discussed later.

CD spectroscopy, reflecting the differential absorption of left-hand circularly and right-hand circularly polarized light, is a useful technique to detect the conformation and configuration of chiral materials. ${ }^{49}$ Here, the CD spectra from UV to visible band are recorded to achieve the complete information about both the surface and the body of chiral NPLs. ${ }^{2,3,20}$ Significantly, the CD spectra in the UV-vis band of asfabricated chiral WZ and ZB CdSe NPLs with the same thickness are distinct along with their similar absorption spectra. In the following parts, we just take L-cysteine-stabilized WZ and ZB NPLs as the representatives for discussion thanks to the wholly mirror symmetry of the CD spectra between Lcysteine- and D-cysteine-stabilized NPLs (Figure 2).
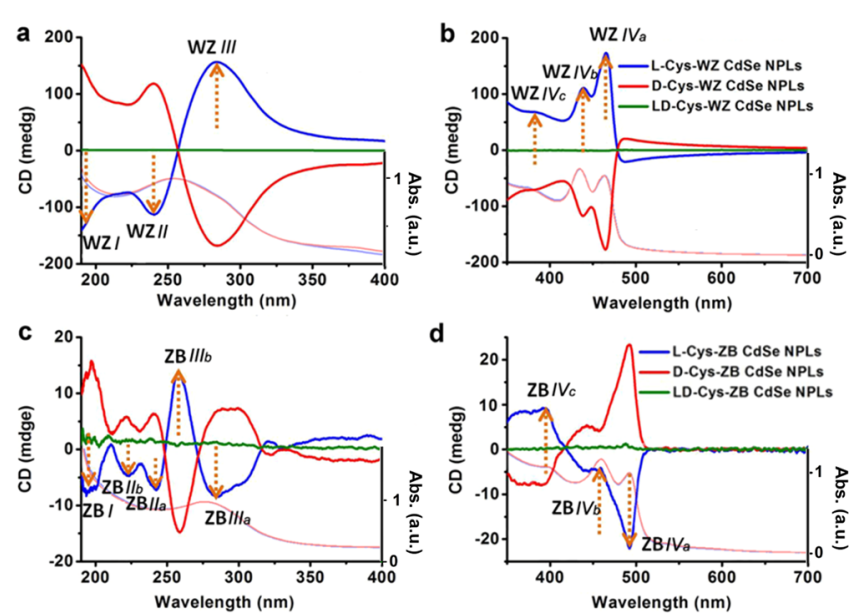

Figure 2. CD spectra of cysteine-stabilized WZ and ZB CdSe NPLs. (a) CD spectra of cysteine stabilized WZ CdSe NPLs in UV band (190-400 nm). (b) CD spectra of cysteine-stabilized WZ CdSe NPLs in the visible band $(350-700 \mathrm{~nm})$. (c) CD spectra of cysteinestabilized ZB CdSe NPLs in the UV band (190-400 nm). (d) CD spectra of cysteine-stabilized ZB CdSe NPLs in the visible band $(350-700 \mathrm{~nm})$. Dotted orange arrows highlight the features of $C D$ peaks.

In the UV region from 190 to $400 \mathrm{~nm}$, the CD spectra of WZ and ZB CdSe NPLs are much more complicated compared with their absorption spectra containing only one broad peak (Figure 2a and 2c). As for L-Cys-WZ CdSe NPLs, the $C D$ spectrum shows three $C D$ peaks, two upward and one downward (blue curve in Figure 2a; peaks WZ I, WZ II, and WZ III are marked by orange dotted arrows). The case for LCys-ZB ones is even more intricate. The CD spectrum displays five peaks, four downward and one upward (blue curve in Figure 2c; peaks ZB I, ZB $I I_{a}, \mathrm{ZB} I I_{b}, \mathrm{ZB} I I I_{a}$, and $\mathrm{ZB} I I I_{b}$ are marked by orange dotted arrows). Obviously, the bonding formation and conformation of L-cysteine on the surface of $\mathrm{ZB}$ NPLs are different than the WZ ones. According to the different binding energy, the electronic transitions of the $\mathrm{CD}$ peaks in UV band are assigned to three groups: (1) the $\mathrm{n}_{\mathrm{O}} \rightarrow$
$\pi_{\mathrm{C}}^{*}=\mathrm{O}$ transition of the $\mathrm{C}=\mathrm{O}$ group in $\mathrm{L}$-cysteine molecules (peaks WZ $I$ and $\mathrm{ZB} \mathrm{I}$ ); ${ }^{50-52}$ (2) the $2 \mathrm{p}_{\mathrm{O}} \rightarrow 5 \mathrm{~s}_{\mathrm{Cd}}$ electronic transition of $\mathrm{Cd}-\mathrm{O}$ bond at the interface between L-cysteine and NPLs (peaks WZ II, ZB $I I_{a}$, and ZB $I_{b}$ ); ${ }^{53}$ and (3) the $3 \mathrm{p}_{\mathrm{S}}$ $\rightarrow 5 s_{\mathrm{Cd}}$ electronic transition of $\mathrm{Cd}-\mathrm{S}$ bond at the interface between L-cysteine and NPLs (peaks WZ III, ZB III $I_{a}$ and ZB $\left.I I I_{b}\right)^{54}$

Unlike the previously reported chiral QDs and QRs with complicated line shape due to splitting of excitonic transitions, ${ }^{20,55}$ the $\mathrm{CD}$ peaks of WZ and ZB NPLs in the visible band from 400 to $700 \mathrm{~nm}$ are simple and have an explicit correspondence with the three absorption peaks (Figure 2b,d). Noteworthily, the $C D$ spectra are distinct between WZ and ZB NPLs. With respect to L-Cys-WZ CdSe NPLs, the CD spectrum shows three upward peaks (blue curve in Figure 2b; peaks WZ $I V_{a}, \mathrm{WZ} I V_{b}$, and $\mathrm{WZ} I V_{c}$ are marked by orange dotted arrows). The peak intensity follows the order of WZ $I V_{a}>\mathrm{WZ} I V_{b}>\mathrm{WZ} I V_{c}$. However, the things are much different in L-Cys-ZB CdSe NPLs. Their CD spectrum displays one downward peak and two upward peaks (blue curve in Figure 2d; peaks $\mathrm{ZB} I V_{a}, \mathrm{ZB} I V_{b}$, and $\mathrm{ZB} I V_{c}$ are marked by orange dotted arrows). $\mathrm{ZB} I V_{a}$ is opposite with $\mathrm{ZB} I V_{b}$ and $\mathrm{ZB}$ $I V_{c}$. Furthermore, the peaks corresponding to the first excitonic transition of WZ and ZB NPLs are opposite (the upward WZ $I V_{a}$ against the downward $\mathrm{ZB} I V_{a}$ ), and the $\mathrm{CD}$ intensity of $\mathrm{ZB}$ $\mathrm{CdSe}$ NPLs is eight times lower than that of WZ ones.

The optical activity of $2 \mathrm{D}$ nanomaterials such as graphene QDs ${ }^{16}$ and $\mathrm{MoS}_{2}{ }^{5}$ has been reported to originate from the preferential structural folding caused by chiral cysteine stabilizers. Here, we offer another insight based on the famous two-group models derived from quantum mechanics. ${ }^{56}$ The reason why choose this model is shown in Part 3 of the Supporting Information. The L-cysteine-stabilized CdSe NPLs are treated as a huge artificial chiral "molecule". The optical activity of this system comes from the surface chiral molecules coupling with NPLs that destroy the intrinsic symmetry of NPLs and endow the whole hybrid optical activity. Therefore, the $\mathrm{C}=\mathrm{O}$ group, $\mathrm{Cd}-\mathrm{O}$ bond, $\mathrm{Cd}-\mathrm{S}$ bond, and CdSe NPL are considered as four chromophores in this huge chiral "molecule", respectively. The asymmetric carbon center in L-cysteine provides a chiral geometric locus among the chromophores.

According to above assumption, the diverse optical activity of L-cysteine-stabilized $\mathrm{WZ}$ and $\mathrm{ZB}$ CdSe NPLs can be described by the nondegenerate coupled-oscillator (NDCO) model, which is the typical one among the two-group models (Figure 3, Part S4 in the Supporting Information). In this model, the CD signal results from coupling of the electric dipole transition moments among the chromophores (Figure 3 ) in a chiral molecule. The CD intensity of chromophore A

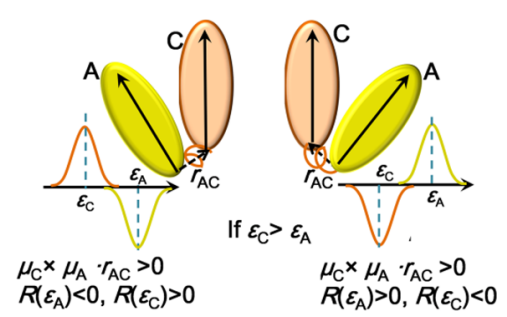

Figure 3. Schematic diagrams of NDCO model with only two chromophores, in which coupling of the dipoles brings out the opposite CD signs. 
depends on the summation of its coupling with other chromophores $\mathrm{C}$, which is expressed as: ${ }^{11}$

$$
R(|00\rangle \rightarrow|10\rangle)=\sum_{\mathrm{C}} \frac{-\varepsilon_{\mathrm{A}} \varepsilon_{\mathrm{C}} V^{\mathrm{lc}}}{\hbar\left(\mathcal{E}_{\mathrm{C}}^{2}-\mathcal{E}_{\mathrm{A}}^{2}\right)}\left(\boldsymbol{\mu}_{\mathrm{C}}^{0 \mathrm{c}} \times \boldsymbol{\mu}_{\mathrm{A}}^{01} \times \boldsymbol{r}_{\mathrm{AC}}\right)
$$

where $R$ is the $\mathrm{CD}$ intensity of chromophore $\mathrm{A} ; \varepsilon_{\mathrm{A}}$ and $\varepsilon_{\mathrm{C}}$ are the transition energies of $\mathrm{A}$ and $\mathrm{C}$, respectively; $V^{\mathrm{lc}}$ arises from the columbic interaction between $\mathrm{A}$ and $\mathrm{C}$ under the light (eq S5); $\hbar$ is the reduced Planck constant; the ground and excited states of A (or C) are marked as 0 and 1 (or 0 and c); $\boldsymbol{\mu}_{\mathrm{A}}$ and $\boldsymbol{\mu}_{\mathrm{C}}$ are the electric dipole transition moments of $\mathrm{A}$ and $\mathrm{C}$, respectively; and the distance vector from $\mathrm{A}$ to $\mathrm{C}$ is denoted as $r_{\mathrm{AC}}$. In this formula, the boldface indicates vector, and the sum represents all of the chromophores $\mathrm{C}$ around A. Clearly, eq 1 reveals that the upward or downward $\mathrm{CD}$ sign comes from the relative location of the coupled chromophores. Figure 3 is a simplified diagram presenting the signs of the $C D$ peaks between two coupling chromophores, whereas the $\mathrm{CD}$ sign induced from chromophore $\mathrm{A}$ to $\mathrm{C}$ is opposite that from $\mathrm{C}$ to A.

Besides, the anisotropic $g$-factor is approximately treated as: $^{57}$

$$
g \cong \frac{4 R(|00\rangle \rightarrow|10\rangle)}{D(|0\rangle \rightarrow|1\rangle)}
$$

here, the electric dipole intensity $D$ equals to the dot product of $\boldsymbol{\mu}_{\mathrm{A}}^{01}$ and $\boldsymbol{\mu}_{\mathrm{A}}^{10}$.

Obviously, above theoretic model indicates that the configuration of surface chiral molecule is a key factor for the optical activity of this system, because the sign of all of the peaks is determined by the configuration of the surface Lcysteine. Therefore, the first problem that needs to be resolved is acquiring a rational configuration of $\mathrm{L}$-cysteine on the surface of WZ and ZB CdSe NPLs. Then, according to the NDCO model, with the help of CD spectra in the UV band, we assume that L-cysteine connects with WZ or ZB CdSe NPLs by $\mathrm{Cd}-\mathrm{O}$ bond (peak WZ II in Figure 2a or peaks ZB $I I_{a}$ and ZB $I_{b}$ in Figure $2 \mathrm{c}$ ) and $\mathrm{Cd}-\mathrm{S}$ bond (peak WZ III in Figure 2a or peaks $\mathrm{ZB} I I I_{a}$ and $\mathrm{ZB} I I_{b}$ in Figure $2 \mathrm{c}$ ). The distinct $\mathrm{Cd}-\mathrm{O}$ and $\mathrm{Cd}-$ $\mathrm{S}$ bonding situation reflected by the $\mathrm{CD}$ spectra in $\mathrm{WZ}$ and $\mathrm{ZB}$ NPLs originates from the different crystal surfaces of WZ and ZB NPLs (Figure 4a-d). The surface of WZ CdSe NPLs is $(11 \overline{2} 0)$ with the "S" shaped alternation of $\mathrm{Cd}$ and Se atoms (Figure 4a). One surface Cd atom connects with two surface Se atoms and one underlying Se atom; hence, it needs only one bond to be a tetrahedron (right inset in Figure 4a). In addition, the Se atoms on the surface of WZ NPLs are easily oxidized in polar solution, leading to the rather-low stability of L- or D-CysWZ CdSe NPLs in water. Thus, the CD study of WZ NPLs should be performed in a no-oxygen circumstance. As a comparison, the crystal surface of ZB CdSe NPLs is (100) and the exposed surface contains only Cd atoms (Figure $4 \mathbf{b}, \mathrm{d}$ ), where each $\mathrm{Cd}$ atom connects with two underlying Se atoms and needs two bonds to form a tetrahedron (right inset in Figure $4 \mathrm{~b}){ }^{37,58}$ Therefore, the surface $\mathrm{Cd}$ atom is easy to bridge the $\mathrm{S}$ atom of cysteine to decrease the dangling bonds. Armed with the above analysis, we propose the possible configurations of $\mathrm{L}$-cysteine on the surface of $\mathrm{WZ}$ and $\mathrm{ZB} \mathrm{CdSe}$ NPLs (Figure 4e,f).

Next, the precise configurations of $\mathrm{L}$-cysteine on the surface of WZ and ZB CdSe NPLs are examined and optimized by

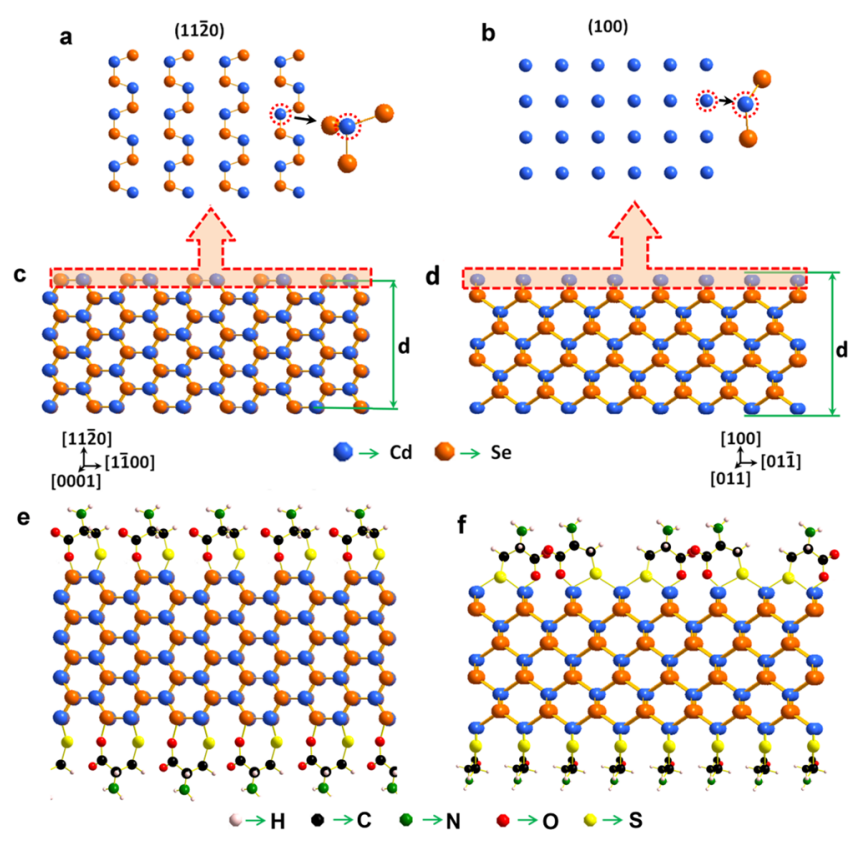

Figure 4. Diagrams of crystal surfaces and structures of $\mathrm{WZ}$ and $\mathrm{ZB}$ CdSe NPLs and the assumptive models for the possible configurations of L-cysteine on the surfaces of WZ and ZB CdSe NPLs. (a) Crystal surface of WZ CdSe NPLs. The right inset presents that one surface $\mathrm{Cd}$ atom connects with two surface $\mathrm{Se}$ atoms and one underlying $\mathrm{Se}$ atom. (b) Crystal surface of ZB CdSe NPLs. The right inset reveals that one surface $\mathrm{Cd}$ atom combines with two underlying Se atoms. (c) Crystal model of WZ CdSe NPLs. (d) Crystal model of ZB CdSe NPLs. (e) Model of possible configuration of L-cysteine on surface of WZ CdSe NPLs. (f) Model of possible configuration of L-cysteine on surface of ZB CdSe NPLs. On surface of WZ and ZB NPLs, L-cysteine is attached with NPLs via the bonds of $\mathrm{Cd}-\mathrm{O}$ and $\mathrm{Cd}-\mathrm{S}$.

DFT (Figure S6). The theoretical thickness of both L-cysteinecapped WZ and ZB NPLs is $2.2 \mathrm{~nm}$ with an assumption of 1.4 $\mathrm{nm}$ thickness for the CdSe crystal layers. This deduction is consistent with the experimental result obtained from atomic force microscope (AFM), i.e., $2.1 \mathrm{~nm}$ for L-Cys-WZ NPL and $2.2 \mathrm{~nm}$ for L-Cys-ZB NPL (Figure S7 and Table S2). Moreover, both DFT and AFM results for the (OAm, OLAm)-WZ CdSe NPLs and OA-ZB CdSe NPLs also support the $1.4 \mathrm{~nm}$ 's thickness of the crystal layers (Figure S7 and Table S2). Note that $1.4 \mathrm{~nm}$ corresponds to 3.5 monolayers (MLs) of WZ NPL and 4.5 MLs of ZB one (Figure S8). After ligand exchange, the thickness of the surface molecule in the WZ NPL system changes from 1.20 to $0.40 \mathrm{~nm}$ (given by DFT, and from 1.25 to $0.35 \mathrm{~nm}$ observed by AFM; Table S2); as for the ZB NPL system, the thickness decreases from 1.10 to $0.4 \mathrm{~nm}$ (given by DFT and from 1.15 to $0.4 \mathrm{~nm}$ discerned by AFM; Table S2). The decreased length of stabilizers and the transfer from the nonpolar or weakly polar solvent (hexane or chloroform) to polar solvent (deionized water) give rise to the red shift of the absorption peaks (Figure 1c,f). The smaller dielectric constant of hexane (1.89) compared to chloroform (4.4) causes the ZB NPLs (in hexane) to have a larger red shift when transferred into water with a dielectric constant of 78.5.

Furthermore, the number of $\mathrm{L}$-cysteine per unit surface of WZ and ZB CdSe NPLs is estimated to be 3 and $8 \mathrm{~nm}^{-2}$ based on the above theoretical model (eqs S44 and S45), which is supported by the thermogravimetric analysis (TGA) (Figure S8 and Table S3). Finally, the X-ray photoelectron spectra (XPS) confirms the formation of $\mathrm{Cd}-\mathrm{O}$ and $\mathrm{Cd}-\mathrm{S}$ bonds in 
WZ and ZB CdSe NPLs, especially the possibility of bridge bonding between $S$ atom and $\mathrm{Cd}$ atom on the surface of $\mathrm{ZB}$ CdSe NPLs (Figures S10 and S11). The bonding energy of the electrons in 1s orbital of $\mathrm{O}$ atom in $\mathrm{Cd}-\mathrm{O}$ bonds is $532.7 \mathrm{eV}$ for L-Cys-WZ NPLs and $532.4 \mathrm{eV}$ for L-Cys-ZB NPLs. The similar value between them implies that the alike $\mathrm{Cd}-\mathrm{O}$ bonds exist in both NPLs. However, the bonding energy of the electrons in $2 \mathrm{p}_{3 / 2}$ orbital of $\mathrm{S}$ atom in $\mathrm{Cd}-\mathrm{S}$ bonds is $162.9 \mathrm{eV}$ in L-Cys-WZ NPLs and $162.1 \mathrm{eV}$ in L-Cys-ZB NPLs, respectively. The lower value in $\mathrm{ZB}$ NPLs suggests the possibility of the $\mathrm{Cd}-\mathrm{S}-\mathrm{Cd}$ bonds. Altogether, the above experimental characterizations validate the proposed pattern of chiral cysteine on the surface of WZ and ZB CdSe NPLs (Figures 4e,f and S6), which lays the basis for the subsequent analysis of CD spectra based on the NDCO model.

In our model, $\mathrm{Cd}-\mathrm{O}$ bond causes the chiral molecule producing a circle on the surface of WZ and ZB CdSe NPLs (Figures 4e,f, S12, and S13). Such a circular configuration of the chiral molecule makes the $C D$ signal of single chromophores arising from the coupling of its electronic transition with all of the other chromophores. For example, the peak WZ $I V_{a}$ in Figure $2 \mathrm{~b}$ results from the summation of coupling of the $1 \mathrm{hh}-1 \mathrm{e}$ transition with the $\mathrm{n}_{\mathrm{O}} \rightarrow \pi_{\mathrm{C}}{ }^{*}=\mathrm{O}$ transition in the $\mathrm{C}=\mathrm{O}$ group, the $2 \mathrm{p}_{\mathrm{O}} \rightarrow 5 \mathrm{~s}_{\mathrm{Cd}}$ transition in the $\mathrm{Cd}-\mathrm{O}$ bond, and the $3 \mathrm{p}_{\mathrm{S}} \rightarrow 5 \mathrm{~s}_{\mathrm{Cd}}$ transition in the $\mathrm{Cd}-\mathrm{S}$ bond (Figure 5a,c). All of the other transitions have a similar origination.
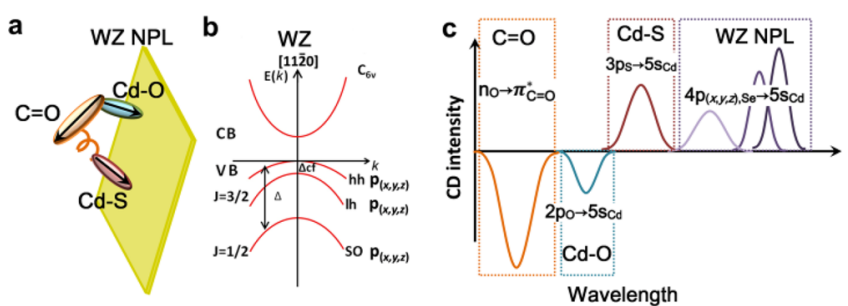

d
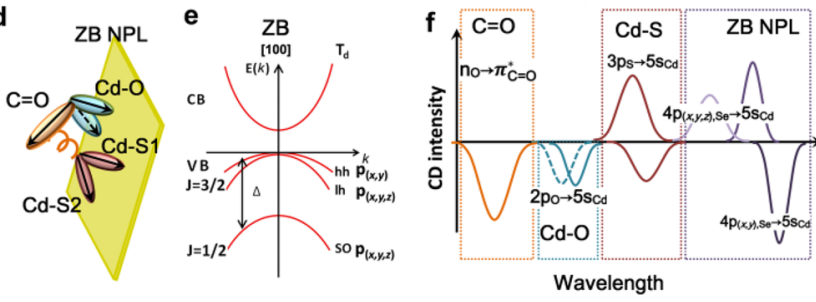

Figure 5. Qualitative analysis for the optical activity of L-cysteinestabilized WZ and ZB CdSe NPL. (a) Simplified view of L-Cys-WZ CdSe NPL. (b) Electronic energy band structure of WZ CdSe along the $[11 \overline{2} 0]$ direction. (c) Charted CD spectrum of L-Cys-WZ CdSe NPLs. (d) Simplified view of L-Cys ZB CdSe NPL. (e) Electronic energy band structure of $\mathrm{ZB} \mathrm{CdSe}$ along the [100] direction. (f) Charted CD spectrum of L-Cys-ZB CdSe NPLs.

Following this concept, the distinct $\mathrm{CD}$ signals from the $\mathrm{Cd}-\mathrm{O}$ bond, the $\mathrm{Cd}-\mathrm{S}$ bond, and the first excitonic transitions of L-Cys-WZ and L-Cys-ZB NPLs can be explored in detail. The first discussed chromophore is the $\mathrm{Cd}-\mathrm{O}$ bond. The CD sign of the transition in $\mathrm{Cd}-\mathrm{O}$ bond is single downward peak (Figure 2a, peak WZ II) in WZ NPLs, while in ZB NPLs, it relates to two downward peaks (Figure 2c, peaks $\mathrm{ZB} I I_{a}$ and $\left.\mathrm{ZB} I_{b}\right)$. The reason is attributed to the number of the possible conformation of L-cysteine on their surface. In the WZ case, only one conformation on the surface induces a single CD peak (Figure S12). However, the oxygen of Lcysteine has two possible bonding positions on the surface of ZB CdSe NPLs to guarantee formation of the circular configuration with minimum energy (Figures $4 \mathrm{f}$ and S13). This leads to two conformations with slightly different $\mathrm{Cd}-\mathrm{O}$ bonding lengths ( 2.3 versus $2.2 \AA)$, dipole moments $(3.40$ versus $3.19 \mathrm{D}$, Table S5), and transition energies (5.57 versus $5.13 \mathrm{eV}$, Table S6). Accordingly, the CD spectrum that is sensitive to the configuration and conformation of the chiral materials would clearly reflect this difference. Furthermore, the two CD peaks are of the same downward tendency owing to their similar relative position with other chromophores, especially with the $\mathrm{Cd}-\mathrm{S}$ bonds that generate the strongest coupling with $\mathrm{Cd}-\mathrm{O}$ due to the closer transition energy and positions compared with other chromophores (Figure S13 and Table S6).

The second chromophore is the $\mathrm{Cd}-\mathrm{S}$ bond. The CD signal of the $\mathrm{Cd}-\mathrm{S}$ bond is one upward peak (Figure 2a, peak WZ III) in L-Cys-WZ NPLs and two opposite peaks (Figure 2c, downward peak ZB $I I I_{a}$ and upward peak ZB $I I_{b}$ ) in L-Cys-ZB NPLs. Only one upward peak, WZ III, manifests the presence of a single $\mathrm{Cd}-\mathrm{S}$ bond between L-cysteine and WZ CdSe NPLs (Figure S12). As a comparison, the two opposite CD peaks $\mathrm{ZB} \mathrm{III}_{a}$ and $\mathrm{ZB} \mathrm{III}_{b}$ in L-Cys-ZB CdSe NPLs reveal the bridge of $\mathrm{Cd}-\mathrm{S}-\mathrm{Cd}$ bonds, where the largely different orientation of the electronic dipoles, the slightly different bonding lengths $(2.58 \AA$ vs $2.57 \AA)$, dipole moments $(2.41$ versus $2.34 \mathrm{D})$ and transition energies (4.81 versus $4.35 \mathrm{eV}$ ) between the two $\mathrm{Cd}-\mathrm{S}$ bonds give two opposite signals (Figure S14 and Table S7).

Then, the excitonic transitions in NPLs are elucidated. We have shown that the $C D$ spectrum at the characteristic absorption of excitonic transitions in QRs reflects their polarization, ${ }^{20}$ which is also true for NPLs. Obviously, the $\mathrm{CD}$ spectra at the excitonic transitions between $\mathrm{WZ}$ and $\mathrm{ZB}$ $\mathrm{CdSe}$ NPLs are much different (Figure $2 \mathrm{~b}, \mathrm{~d}$ ). That is, the polarization of the excitonic transitions in $\mathrm{WZ}$ and $\mathrm{ZB} \mathrm{CdSe}$ NPLs are distinct due to the varied crystal structure and quantum-confinement direction. As for WZ CdSe NPLs, the quantum confinement direction is along the low-symmetry $[11 \overline{2} 0]$ direction. Thereby, the states of the valence band are the mixture of $4 \mathrm{p}_{x}, 4 \mathrm{p}_{y}$, and $4 \mathrm{p}_{z}$; namely, all of the allowed transitions would contain a $4 \mathrm{p}_{(x, y, z), \mathrm{Se}} \rightarrow 5 \mathrm{~s}_{\mathrm{Cd}}$ transition (Figure $5 b, c)$. This is the reason for the three upward $C D$ peaks (Figure $2 \mathrm{~b}, \mathrm{WZ} I V_{a}, \mathrm{WZ} I V_{b}$, and $\mathrm{WZ} I V_{c}$ ) appearing in $\mathrm{WZ}$ CdSe NPLs. It is noticed that the weak and broad peak appears in the long wavelength, which likely comes from the defect state caused by ligand exchange. However, with respect to ZB NPLs, the quantum confinement direction is along [100], so the 1 hh-1e exciton originates from the $4 \mathrm{p}_{(x, y), \mathrm{Se}} \rightarrow 5 \mathrm{~s}_{\mathrm{Cd}}$ transition, while the 1lh-1e exciton results from the $4 \mathrm{p}_{(x, y, z), \mathrm{Se}}$ $\rightarrow 5 s_{\mathrm{Cd}}$ transition (Figure 5e). ${ }^{59}$ As a result, the upward and downward tendencies of CD peaks are opposite between 1hhle and 1lh-1e transitions due to their different polarization transitions. Moreover, because the $1 S \mathrm{O}$ state has the same symmetry with the 1 lh state, the $1 \mathrm{SO}-1 \mathrm{e}$ exciton would show the same transition polarization with $1 \mathrm{lh}-1 \mathrm{e}$ exciton (Figure 5e,f). Therefore, the peaks $\mathrm{ZB} I V_{b}$ and $\mathrm{ZB} I V_{c}$ have the same direction and are opposite to peak $\mathrm{ZB} I V_{a}$ (Figure $2 \mathrm{~d}$ ).

The detailed excitonic transitions contributing to the optical activity of L-cysteine stabilized WZ and ZB CdSe NPLs are sketched out in Figure 6. Obviously, the excitonic transitions in WZ NPLs from low to high energy are in sequence the 

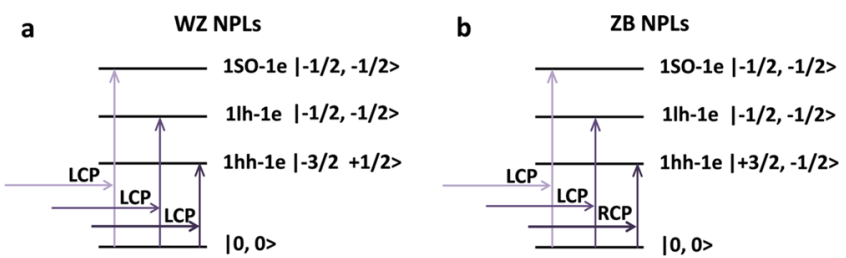

Figure 6. Schematic structure of possible excitonic transitions: (a) 1hh-1e, 1lh-1e, and 1SO-1e exciton transitions in WZ CdSe NPLs. (b) $1 \mathrm{hh}-1 \mathrm{e}, 1 \mathrm{~h}-1 \mathrm{e}$, and 1SO-1e exciton transitions in ZB CdSe NPLs.

transitions from $|0,0\rangle$ to $|-3 / 2,+1 / 2\rangle ;|-1 / 2,-1 / 2\rangle$; and $|-1 / 2,-1 / 2\rangle$ (Figure 6a), corresponding to $1 \mathrm{hh}-1 \mathrm{e}, 1 \mathrm{lh}-1 \mathrm{e}$, and $1 \mathrm{SO}-1 \mathrm{e}$ excitons with absorption of left circularly polarized light (LCP). As for ZB CdSe NPLs, 1hh-1e excitonic transition from $|0,0\rangle$ to $|+3 / 2,-1 / 2\rangle$ is much different with strong absorption of right circular polarized light (RCP) compared with the other two excitonic transitions of $1 \mathrm{lh}-1 \mathrm{e}$ and $1 \mathrm{SO}-1 \mathrm{e}$ from $|0,0\rangle$ to $|-1 / 2,-1 / 2\rangle$ and $|-1 / 2,-1 / 2\rangle$ (Figure $6 b$ ).

One more thing needed to be mentioned is that the CD intensity of the 1 hh-1e excitonic transition in L-Cys-WZ NPLs is eight times stronger than that of ZB NPLs. A pair of main factors influence the CD intensity of WZ and ZB NPLs. One is the dipole moment. The dipole moment in WZ NPLs along [11 20 ] estimated by DFT is $23.5 \mathrm{D}$ that is considerably larger than 8.1 D in ZB NPLs (Table S5). The second is the polarization. The WZ CdSe NPLs have a polarization along the direction of [0001], but the ZB NPLs is isotropic in-plane. The polarization of WZ NPLs increases the perturbation term of dipole-dipole interaction between NPL and chiral molecular (eqs S20 versus S22), consequently enhancing the CD intensity from UV to visible bands (Tables S8 and S9).

Table 1 quantitatively compares the experimental data and the calculated results based on NDCO model. Impressively, it shows excellent agreement.

Table 1. DFT Calculated and Experimental Results of the Anisotropic $\mathrm{g}$-Factor of $\mathrm{Cd}-\mathrm{O}, \mathrm{Cd}-\mathrm{S}$ Bonds, and the First Excitonic Transition in L-Cys-WZ CdSe NPLs and L-Cys-ZB CdSe NPLs

\begin{tabular}{|c|c|c|c|c|}
\hline & & $\begin{array}{l}\text { L-Cys-WZ } \\
\text { NPLs }\end{array}$ & L-Cys-ZB & NPLs \\
\hline \multirow[t]{2}{*}{$g_{\mathrm{Cd}-\mathrm{O}}$} & experimental & $-3.6 \times 10^{-3}$ & $-2.5 \times 10^{-4}$ & $-1.5 \times 10^{-4}$ \\
\hline & theoretical & $-7.8 \times 10^{-3}$ & $-6.3 \times 10^{-5}$ & $-4.6 \times 10^{-5}$ \\
\hline \multirow[t]{2}{*}{$g_{\mathrm{Cd}-\mathrm{S}}$} & experimental & $5.7 \times 10^{-3}$ & $4.2 \times 10^{-4}$ & $-2.7 \times 10^{-4}$ \\
\hline & theoretical & $3.9 \times 10^{-3}$ & $1.9 \times 10^{-4}$ & $-1.4 \times 10^{-4}$ \\
\hline \multirow[t]{2}{*}{$g_{\mathrm{NPL}}$} & experimental & $5.3 \times 10^{-3}$ & $-6.7 \times 10^{-4}$ & \\
\hline & theoretical & $9.8 \times 10^{-3}$ & $-2.7 \times 10^{-4}$ & \\
\hline
\end{tabular}

Finally, the optical activity of this system is adjustable by controlling the thickness of WZ and ZB CdSe NPLs (Figures S15 and S16 and Table S4). The increase of the thickness has little influence on the shape of the CD spectrum. Furthermore, the crystal-structure-dependent optical activity of the CdSe NPLs is also found with other chiral inducers (Figure S17).

It must be pointed out that the two-group model adopted here does not involve hybridization of the delocalized quasiparticle states with the molecular states, which has been considered in previous TDDFT calculations. ${ }^{14,17}$ In future study, that additional effect of the chiral molecules' state on the optical activity of the whole system might need to be included, which will make our understanding about the optical activity of chiral NCs more accurate.

In summary, we demonstrate that $\mathrm{CD}$ spectroscopy is a powerful tool for exploring the optical properties of semiconductor nanomaterials covered by chiral molecules on their surfaces. Taking L- or D-cysteine-stabilized WZ and ZB CdSe NPLs with $1.4 \mathrm{~nm}$ thickness as example, we show that the measured CD spectrum provides the fine information on both the surface configuration and excitonic transitions. The distinct configuration of the chiral cysteine on the surface of $\mathrm{WZ}$ and ZB CdSe NPLs found in the CD spectra is verified by the experimental and theoretical data from AFM, TGA, and XPS measurements and DFT calculations. Furthermore, the insights are provided to understand how the CD spectrum depends on the crystal structure, NPL orientation, and nanocrystal size. This research thus paves the avenue toward application of the CD spectrum in the field of chiral nanomaterials, which will be an important additive to chiral chemistry, nanoscience, and optical physics.

\section{ASSOCIATED CONTENT}

\section{S Supporting Information}

The Supporting Information is available free of charge on the ACS Publications website at DOI: 10.1021/acs.nanolett.8b01001.

Experimental results, theoretical calculations, and additional figures (PDF)

\section{AUTHOR INFORMATION}

\section{Corresponding Author}

*E-mail: zytang@nanoctr.cn; phone: +86-010-82545580.

ORCID ${ }^{\circ}$

Xiuwen Zhang: 0000-0003-1003-2885

Zhiyong Tang: 0000-0003-0610-0064

Notes

The authors declare no competing financial interest.

\section{ACKNOWLEDGMENTS}

The authors acknowledge financial support from National Key Basic Research Program of China (grant nos. 2014CB931801 and 2016YFA0200700 to Z.Y.T. and 2016YFB0700700 to S.H.W.), National Natural Science Foundation of China (grant nos. 21805188 to X.Q.G.; 21721002 and 21475029 to Z.Y.T.; 11774239 to X.W.Z; 51672023, 11634003, and U1530401 to S.H.W.; and 11804230 to P.H), Frontier Science Key Project of Chinese Academy of Sciences (grant no. QYZDJ-SSWSLH038 to Z.Y.T.), K. C. Wong Education Foundation (Z.Y.T.), and Shenzhen Science and Technology Innovation Commission (grant nos. JCYJ20170818093035338, JCYJ20170412110137562, and ZDSYS201707271554071 to X.W.Z). We thank Prof. Jingbo Li for useful discussion, Mr. Zhangzhang Lei for computation of the data, and Ms. Xiao He for AFM characterization.

\section{REFERENCES}

(1) Moloney, M. P.; Gun'ko, Y. K.; Kelly, J. M. Chem. Commun. 2007, 3900-3902.

(2) Zhou, Y.; Yang, M.; Sun, K.; Tang, Z.; Kotov, N. A. J. Am. Chem. Soc. 2010, 132, 6006-6013.

(3) Ben Moshe, A.; Szwarcman, D.; Markovich, G. ACS Nano 2011, 5, 9034-9043. 
(4) Zhou, Y.; Zhu, Z.; Huang, W.; Liu, W.; Wu, S.; Liu, X.; Gao, Y.; Zhang, W.; Tang, Z. Angew. Chem., Int. Ed. 2011, 50, 11456-11459. (5) Purcell-Milton, F.; McKenna, R.; Brennan, L. J.; Cullen, C. P.; Guillemeney, L.; Tepliakov, N. V.; Baimuratov, A. S.; Rukhlenko, I. D.; Perova, T. S.; Duesberg, G. S.; Baranov, A. V.; Fedorov, A. V.; Gun'ko, Y. K. ACS Nano 2018, 12, 954-964.

(6) Huo, S.; Duan, P.; Jiao, T.; Peng, Q.; Liu, M. Angew. Chem., Int. Ed. 2017, 56, 12174-12178.

(7) Wang, Y.; Xu, J.; Wang, Y.; Chen, H. Chem. Soc. Rev. 2013, 42, 2930-2962.

(8) Ben-Moshe, A.; Maoz, B. M.; Govorov, A. O.; Markovich, G. Chem. Soc. Rev. 2013, 42, 7028-7041.

(9) Ma, W.; Xu, L.; de Moura, A. F.; Wu, X.; Kuang, H.; Xu, C.; Kotov, N. A. Chem. Rev. 2017, 117, 8041-8093.

(10) Yeom, J.; Santos, U. S.; Chekini, M.; Cha, M.; de Moura, A. F.; Kotov, N. A. Science 2018, 359, 309-314.

(11) Nordén, B.; Rodger, A.; Dafforn, T. Linear Dichroism and Circular Dichroism: A Textbook on Polarized-Light Spectroscopy; Royal Society of Chemistry: Cambridge, U.K., 2010.

(12) Nakashima, T.; Kobayashi, Y.; Kawai, T. J. Am. Chem. Soc. 2009, 131, 10342-10343.

(13) Govan, J. E.; Jan, E.; Querejeta, A.; Kotov, N. A.; Gun'ko, Y. K. Chem. Commun. (Cambridge, U. K.) 2010, 46, 6072-6074.

(14) Elliott, S. D.; Moloney, M. c. 1. P.; Gun'ko, Y. K. Nano Lett. 2008, 8, 2452-2457.

(15) Choi, J. K.; Haynie, B. E.; Tohgha, U.; Pap, L.; Elliott, K. W.; Leonard, B. M.; Dzyuba, S. V.; Varga, K.; Kubelka, J.; Balaz, M. ACS Nano 2016, 10, 3809-3815.

(16) Suzuki, N.; Wang, Y.; Elvati, P.; Qu, Z.-B.; Kim, K.; Jiang, S.; Baumeister, E.; Lee, J.; Yeom, B.; Bahng, J. H.; Lee, J.; Violi, A.; Kotov, N. A. ACS Nano 2016, 10, 1744-1755.

(17) Tohgha, U.; Deol, K. K.; Porter, A. G.; Bartko, S. G.; Choi, J. K.; Leonard, B. M.; Varga, K.; Kubelka, J.; Muller, G.; Balaz, M. ACS Nano 2013, 7, 11094-11102.

(18) Grimme, S. J. Chem. Phys. 2013, 138, 244104.

(19) Risthaus, T.; Hansen, A.; Grimme, S. Phys. Chem. Chem. Phys. 2014, 16, 14408-14419.

(20) Gao, X.; Zhang, X.; Deng, K.; Han, B.; Zhao, L.; Wu, M.; Shi, L.; Lv, J.; Tang, Z. J. Am. Chem. Soc. 2017, 139, 8734-8739.

(21) Delgado-Pérez, T.; Bouchet, L. M.; de la Guardia, M.; Galian, R. E.; Pérez-Prieto, J. Chem. - Eur. J. 2013, 19, 11068-11076.

(22) Han, C.; Li, H. Small 2008, 4, 1344-1350.

(23) Wei, Y.; Li, H.; Hao, H.; Chen, Y.; Dong, C.; Wang, G. Polym. Chem. 2015, 6, 591-598.

(24) Wei, Y.; Hao, H.; Zhang, J.; Hao, X.; Dong, C. Anal. Methods 2014, 6, 3227-3230.

(25) Mukhina, M. V.; Korsakov, I. V.; Maslov, V. G.; Purcell-Milton, F.; Govan, J.; Baranov, A. V.; Fedorov, A. V.; Gun'ko, Y. K. Sci. Rep. 2016, 6, 24177.

(26) Liu, W.; Choi, H. S.; Zimmer, J. P.; Tanaka, E.; Frangioni, J. V.; Bawendi, M. J. Am. Chem. Soc. 2007, 129, 14530-14531.

(27) Pinaud, F.; King, D.; Moore, H.-P.; Weiss, S. J. Am. Chem. Soc. 2004, 126, 6115-6123.

(28) Li, Y.; Zhou, Y.; Wang, H. Y.; Perrett, S.; Zhao, Y.; Tang, Z.; Nie, G. Angew. Chem., Int. Ed. 2011, 50, 5860-5864.

(29) Shah, E.; Soni, H. P. RSC Adv. 2013, 3, 17453-17461.

(30) Vulugundam, G.; Misra, S. K.; Ostadhossein, F.; SchwartzDuval, A. S.; Daza, E. A.; Pan, D. Chem. Commun. 2016, 52, 75137516.

(31) Tedsana, W.; Tuntulani, T.; Ngeontae, W. Anal. Chim. Acta 2015, $867,1-8$

(32) Bloom, B. P.; Kiran, V.; Varade, V.; Naaman, R.; Waldeck, D. H. Nano Lett. 2016, 16, 4583-4589.

(33) Bloom, B. P.; Graff, B. M.; Ghosh, S.; Beratan, D. N.; Waldeck,

D. H. J. Am. Chem. Soc. 2017, 139, 9038-9043.

(34) Alivisatos, A. P. Science 1996, 271, 933-937.

(35) Subila, K. B.; Kishore Kumar, G.; Shivaprasad, S. M.; George Thomas, K. J. Phys. Chem. Lett. 2013, 4, 2774-2779.
(36) Wang, F.; Wang, Y.; Liu, Y.-H.; Morrison, P. J.; Loomis, R. A.; Buhro, W. E. Acc. Chem. Res. 2015, 48, 13-21.

(37) Ithurria, S.; Dubertret, B. J. Am. Chem. Soc. 2008, 130, 1650416505 .

(38) Son, J. S.; Wen, X.-D.; Joo, J.; Chae, J.; Baek, S.-i.; Park, K.; Kim, J. H.; An, K.; Yu, J. H.; Kwon, S. G.; Choi, S.-H.; Wang, Z.; Kim, Y.-W.; Kuk, Y.; Hoffmann, R.; Hyeon, T. Angew. Chem., Int. Ed. 2009, $48,6861-6864$.

(39) Naeem, A.; Masia, F.; Christodoulou, S.; Moreels, I.; Borri, P.; Langbein, W. Phys. Rev. B: Condens. Matter Mater. Phys. 2015, 91, 121302.

(40) Grim, J. Q.; Christodoulou, S.; Di Stasio, F.; Krahne, R.; Cingolani, R.; Manna, L.; Moreels, I. Nat. Nanotechnol. 2014, 9, 891895.

(41) Scott, R.; Achtstein, A. W.; Prudnikau, A.; Antanovich, A.; Christodoulou, S.; Moreels, I.; Artemyev, M.; Woggon, U. Nano Lett. 2015, 15, 4985-4992.

(42) Rowland, C. E.; Fedin, I.; Zhang, H.; Gray, S. K.; Govorov, A. O.; Talapin, D. V.; Schaller, R. D. Nat. Mater. 2015, 14, 484.

(43) Dong, S.; Lian, J.; Jhon, M. H.; Chan, Y.; Loh, Z.-H. Nano Lett. 2017, 17, 3312-3319.

(44) Scott, R.; Heckmann, J.; Prudnikau, A. V.; Antanovich, A.; Mikhailov, A.; Owschimikow, N.; Artemyev, M.; Climente, J. I.; Woggon, U.; Grosse, N. B.; Achtstein, A. W. Nat. Nanotechnol. 2017, $12,1155-1160$

(45) Achtstein, A. W.; Antanovich, A.; Prudnikau, A.; Scott, R.; Woggon, U.; Artemyev, M. J. Phys. Chem. C 2015, 119, 2015620161 .

(46) Li, L.-s.; Hu, J.; Yang, W.; Alivisatos, A. P. Nano Lett. 2001, 1, 349-351.

(47) Son, J. S.; Yu, J. H.; Kwon, S. G.; Lee, J.; Joo, J.; Hyeon, T. Adv. Mater. 2011, 23, 3214-3219.

(48) Yeh, C.-Y.; Wei, S.-H.; Zunger, A. Phys. Rev. B: Condens. Matter Mater. Phys. 1994, 50, 2715-2718.

(49) Berova, N.; Bari, L. D.; Pescitelli, G. Chem. Soc. Rev. 2007, 36, 914-931.

(50) Osted, A.; Kongsted, J.; Christiansen, O. J. Phys. Chem. A 2005, 109, 1430-1440.

(51) Maul, R.; Preuss, M.; Ortmann, F.; Hannewald, K.; Bechstedt, F. J. Phys. Chem. A 2007, 111, 4370-4377.

(52) Nishino, H.; Kosaka, A.; Hembury, G. A.; Aoki, F.; Miyauchi, K.; Shitomi, H.; Onuki, H.; Inoue, Y. J. Am. Chem. Soc. 2002, 124, $11618-11627$.

(53) Schleife, A.; Fuchs, F.; Furthmüller, J.; Bechstedt, F. Phys. Rev. B: Condens. Matter Mater. Phys. 2006, 73, 245212.

(54) Willner, H.; Vasak, M.; Kaegi, J. H. R. Biochemistry 1987, 26, 6287-6292.

(55) Ben-Moshe, A.; Teitelboim, A.; Oron, D.; Markovich, G. Nano Lett. 2016, 16, 7467-7473.

(56) Craig, D. P.; Thirunamachandran, T. Molecular quantum electrodynamics: an introduction to radiation-molecule interactions; Courier Corporation: North Chelmsford, MA, 1984.

(57) Schellman, J. A. Chem. Rev. 1975, 75, 323-331.

(58) Li, Z.; Peng, X. J. Am. Chem. Soc. 2011, 133, 6578-6586.

(59) Heckmann, J.; Scott, R.; Prudnikau, A. V.; Antanovich, A.; Owschimikow, N.; Artemyev, M.; Climente, J. I.; Woggon, U.; Grosse, N. B.; Achtstein, A. W. Nano Lett. 2017, 17, 6321-6329. 